\title{
Inside a physicist
}

\section{John Stachel}

H.A. Kramers: Between Tradition and Revolution. By M. Dresden. SpringerVerlag:1987. Pp.563. DM 120,\$74.95, £45.

ThE Dutch theoretical physicist Hendrik Antonie Kramers is probably known to more physicists as an initial than as a name. How many realize what lies hidden behind the modest ' $\mathrm{K}$ ' of the WKBJ approximation method in quantum mechanics? A smaller number will be familiar with one or another of such phrases as Kramers theorem or the Kramers-Kronig relations, but his name is hardly a household word among the current generation of physicists. Yet, as major factors leading Kramers to turn away from active participation in work of the quartet just when it was about to culminate in the definitive formulation of the new mechanics.

Kramers left Copenhagen in 1926 to accept the chair of physics in Utrecht, becoming the dean of the Dutch physics community after the deaths of Lorentz and Ehrenfest. His life and scientific activity after the move are treated sketchily in Part Three, except for a chapter of over 100 pages devoted to his work on quantum electrodynamics. Like Pauli, Kramers was very critical of Dirac's approach. He felt that the answer to the problems of the quantum theory lay in a careful reexamination of the classical theory of the interaction of electrons and radiation. Dresden shows that his faithfulness to the classical legacy of Lorentz's electron of its author in several ways. Dresden is a distinguished physicist from Holland, who studied with Kramers before going to the United States in 1939, and who met him again several times after the war. $\mathrm{He}$ is clearly very involved, both intellectually and emotionally, with the subject and his milieu (he spent 11 years working on this book), and is well acquainted with many others who figure in the story. The book is based on a close reading of Kramers's writings, both technical and popular, and of a host of other sources in physics and the history of physics. As might be expected from a physicist of Dresden's eminence, the explanations of Kramers's work are masterly and will be indispensable for those interested in the early history of quantum mechanics and of quantum electrodynamics. But Dresden attempts much more than exposition. $\mathrm{He}$

\section{IMAGE UNAVAILABLE FOR COPYRIGHT REASONS}

Kramers and contemporaries - the famous line-up at the Fifth Solvay Conference in 1927. Kramers is fourth from the left in the second row.

Max Dresden sets out to demonstrate, he has a claim to be remembered along with that distinguished trio consisting of his friends, Wolfgang Pauli and Werner Heisenberg, and his mentor, Niels Bohr, who contributed so decisively in the early 1920 s to shaping the emerging quantum mechanics, and through it the course of physics over the past 60 years.

The depiction of the interrelationships between Kramers and the other members of this quartet, and a masterly discussion of some of its consequences for Kramers's research, form the central core of the book. After some introductory scientific background in the first four chapters, the remainder of Part 1 gives a quick introduction to the quartet and a summary of their accomplishments during the decade $(1916-1926)$ that Kramers was in Copenhagen as Bohr's assistant. The second part is a detailed survey of Kramers's work during this period, which culminate in two rewarding but technically demanding chapters on the Bohr-Kramers-Slater (BKS) theory and the Kramers-Heisenberg dispersion theory. The author establishes the importance of Kramers in the events that led Heisenberg to formulate matrix mechanics. He suggests that Kramers's depression after the collapse of the BKS theory, in which he had placed such exaggerated hopes, as well as fear of competition with Heisenberg, to whom Kramers felt intellectually inferior, were theory was a source of both strengths and weaknesses in Kramers's work. After the Second World War, Kramers's approach, with its emphasis on the need for mass renormalization, started to receive widespread recognition among physicists searching for a way to understand the newly discovered Lamb shift. But Kramers again turned away from active participation in the further development of the theory that led to the now classic Feynman-Schwinger-Dyson relativistic version of quantum electrodynamics.

In the final part of the book, Dresden attempts to assess Kramers's personality and scientific style, his social and religious commitments (or lack of them), and his self-image. A lot of additional biographical material is presented here, so that a reader trying to form a connected picture of Kramers's life has to do a good deal of skipping around. The author explains that he was forced to cut down a manuscript of some 1,400 printed pages, which may account for certain imbalances and gaps in the treatment of various periods in Kramers's life, as well as the scattering of information on some topics throughout the book. (The circumstances of Kramers's marriage, for example, are discussed at several widely separated places.) The index is very weak and does not make it any easier to gather together such information.

This biography bears the distinct stamp tries to explain the intra-psychic reasons for the succession of 'near misses' in Kramers's career - cases in which he just failed to make some major discovery. He also speculates on why Kramers never worked on certain problems that he was eminently qualified to tackle.

These portions of the book lean very heavily on a number of personal interviews with members of Kramers's family, close friends and fellow scientists, who would presumably not have spoken so freely to someone in whom they did not have full confidence. Kramers's children made available his diaries, date books and private poems. This material enables Dresden to provide a number of remarkable insights into his subject's inner life.

The picture that he presents is a gloomy one. Dresden sees an essential continuity between certain basic traits manifested in Kramers's closest interpersonal relations, in his relations with his fellow scientists and even in his relationship to science itself. An inability to commit himself completely to anyone, friend or wife, or to any cause, religious or political, showed itself early in his life and deepened as he grew older. He feared any major decision, because it foreclosed all alternative paths. His strong attraction to and talent for such varied fields as music, literature and science made the choice of a career particularly difficult for the young man. His first mentor, Paul Ehrenfest, felt that this 
trait would prevent him from succeeding in physics. Kramers also had a horror of interpersonal conflicts, and would go to extraordinary lengths to avoid them. He regarded this as a family characteristic, telling a young relative "A Kramers has to fight to fight". He was well aware that his inability to fight for his convictions hindered him scientifically.

Perhaps the most remarkable example is Kramers's unpublished anticipation of the Compton effect. The idea occurred to him in 1921, but he allowed Bohr, who at the time was a vigorous opponent of the photon concept, to talk him out of publishing his work. Indeed, Bohr was so effective in converting Kramers to his point of view that Kramers became an even more intemperate opponent of the photon than Bohr himself. Compton later received a Nobel prize for his work, a prize which it is reasonable to suppose Kramers would have shared had he predicted the effect two years before the experiment. It is sad to learn that Kramers's life, like that of many other outstanding scientists, was embittered by his failure ever to receive a coveted Nobel. One is left with the impression of a brilliant, upright and conscientious man, torn by incessant internal conflicts, constantly tormented by feelings of personal and professional lack of fulfilment, who maintained external control over his feelings at a great cost to himself.

How accurate is this picture? It is hard for an outsider to know. Dresden freely admits that the book gives " $m y$ understanding of Kramers' life". The wealth of intimate detail about Kramers that Dresden provides relies so heavily on personal interviews (Dresden himself notes the " 'soft' character" of this information) that it is difficult for others to assess the evidence until the interviews (which I hope were taped), as well as Kramers's personal papers, are made available to others. Information that would seem important to an assessment of Kramers's psychological development is not always provided. (His father turns up remarried when Kramers is in his midtwenties, for example, without any indication of what happened to his mother.)

We must be immensely grateful to Dresden for having broken with the genteel tradition of scientific biography by writing a fascinating book that leads us into the forbidden territory of Kramers's personal and psychic life. However, one sometimes feels the need for a more expert guide through this territory, the sort of superlative guide that Dresden proves to be through the world of Kramers's physics.

John Stachel, 745 Commonwealth Avenue, Boston, Massachusetts 02215, USA, is Profes sor of Physics at Boston University and Editor of the Collected Papers of Albert Einstein, the first volume of which appeared in 1987.

\section{Jump in quantum history}

\author{
Nicholas Kemmer
}

The Historical Development of Quantum Theory. Vol. 5 Erwin Schrödinger and the Rise of Wave Mechanics. Part 1 Schrödinger in Vienna and Zürich 18871925; Part 2 The Creation of Wave Mechanics: Early Response and Applications 1925-1926. By Jagdish Mehra and Helmut Rechenberg. Springer-Verlag: 1987. Pp.980. Part 1 DM98, £36, \$54. Part 2 DM148, £55, \$79.95.

QuANTUM theory was born with this century and needed a quarter of it to reach maturity as quantum mechanics. That theory soon gained almost universal acceptance as the key to a much-deepened understanding of inanimate nature.

As Jagdish Mehra told us at the start of this multi-volume work, he began preparing it just about another quarter-century later. For him, the time was then most auspicious; nearly all those who, from 1925 onwards had taken part in creating and developing quantum mechanics, were still active, not to mention some of their teachers. Mehra established happy contacts with, it would seem, nearly all these people. A large part of the work that Mehra then began is now in print. Since 1970 he has had Helmut Rechenberg as partner.

Mehra displays a special ability to convert copious records of formal interviews and informal exchanges into lively accounts that ring very true. These are not just incidental but rather quite central to the structure of the first four volumes of the work, which are not strictly chronological in order. They split into parts presenting the ideas that relate roughly to the work of Planck, Bohr, Pauli, Heisenberg and Dirac. Embedded into the very readable technical sections there are biographies and pen pictures of these men and descriptions of their backgrounds. Naturally the work of many other people is described, some of whom receive similar, though shorter treatment. Also, as an introduction, figures from the prehistory of quantum theory appear in the setting of their achievements, with very detailed documentation for each one.

The reader-interest of different sections of these volumes inevitably varies. For me, the later technical chapters deal with topics and publications I remember from long ago, while earlier parts invite study for the twists and turns of how it was before my time. As for the vast amount of background information and biographical detail, one must be grateful that it exists.

Now, after a five-year gap, a further two-part volume of this work has been published. It is dedicated to Erwin Schrödinger, and appears at a time when the centenary of his birth has just been celebrated. Schrödinger was undoubtedly a great physicist and a remarkable person. Why, however, was he not covered together with the 'greats' of the previous volumes? That would have been appropriate chronologically - and indeed his name is not absent from those earlier pages. Schrödinger receives special treatment because, within the history of quantum theory, he holds a quite unusual position. He was a most distinguished scientist who worked on many aspects of theoretical physics, but until 1926 his contributions to quantum theory were quite modest. In that year he published a series of papers that immediately transformed ideas and methods in quantum theory quite profoundly. In due course this work gained him a Nobel prize. However, because the interpretation of his 'wave mechanics' by the great majority of his colleagues differed drastically from his own, he soon parted company with them. Only his wave equation flourished within quantum mechanics.

This volume is clearly meant both to continue the series and to tell Schrödinger's story. Given the authors' general intent to provide maximum background material, it could hardly be the former without the latter. There was thus a great amount of further background material to collect beyond Mehra's earlier work (which had included interviews with Schrödinger), explaining the time gap between the appearance of the previous volumes and this one. Schrödinger left a huge amount of archival material behind which the authors not only use but also list in detail, and in addition much has been assembled on his Viennese background and some on Zürich, where his irruption into quantum theory took place.

Description of Vienna in the late nineteenth century begins the work. We are told some history of the multinational Habsburg Empire and are introduced to its many-sided culture, including a number of its great figures. Schrödinger enters as an inheritor of great traditions - a polymath growing up in a highly cultured family. Every event, place or person mentioned is the subject of detailed documentation.

The reader learns of many men who influenced Schrödinger in his youth. Perhaps the most relevant of them is Ludwig Boltzmann, because statistical mechanics became one of Schrödinger's main interests. (Boltzmann is first introduced alongside Ernst Mach, and we hear incidentally of their great debate on the acceptability of atoms as real.) In 1912 Schrödinger began to be known to theoretical physicists through his prolific publications on many topics. Space forbids discussing them here, as Mehra and Rechenberg do 